\title{
Methodological issues for evaluation of alcohol and other drug effects: Examples from flight-simulator performance
}

\author{
JAMES C. MUNDT \\ Vermont Alcohol Research Center, Colchester, Vermont \\ and \\ LEONARD E. ROSS \\ University of Wisconsin, Madison, Wisconsin
}

\begin{abstract}
Individual differences and idiosyncratic responses to alcohol and other drugs present challenges to researchers concerning how tasks should be structured and performance analyzed. Defined scenarios allow operators to perform known maneuvers over a predetermined time course to the best of their abilities, whereas episodic scenarios embed specific situations into more dynamic, realistic, and interactive testing sessions. Degraded performance in the defined scenarios is easily defined as deviations from a hypothetically perfect performance profile, but assessment of alcohol and other drug effects on individual performances in dynamic situations requires flexible analytic procedures. An example of scoring an episodic flight scenario event using flightsimulator data is given, and an interactive analysis system developed to analyze idiosyncratic pilot responses is described.
\end{abstract}

System simulation makes it possible to investigate the effects of alcohol and other drugs under conditions that would be difficult, if not impossible, to repeat with consistency in actual operational contexts. The fact that simulators can present a variety of situations to system operators and permit complete performance information to be recorded for analysis raises the question of how evaluation situations should be structured to permit accurate examination of the effects of alcohol and other drugs on performance. This is a particularly important issue because of two major evaluation problems. The first concerns the large individual differences evident in the performance of complex dynamic tasks in general, and the second is the manner in which alcohol and other drugs affect performance in complicated tasks such as piloting. With respect to the former, Nolan, Hettinger, Kennedy, and Edinger (1988) have pointed out that individual differences are the major source of variability in studies designed to assess simulator equipment features, even when homogeneous groups of highly experienced system operators are used. Evaluation of the effects of alcohol and other drugs on performance faces a similar problem of individual variability, not only between subjects because of drug-tolerance differences but also from instance to instance with the same subject because of learning and testing order effects.

\footnotetext{
This research was supported by Grant AA6093 from the National Institute on Alcohol Abuse and Alcoholism to L. E. Ross. Correspondence should be addressed to J. C. Mundt, Vermont Alcohol Research Center, 2000 Mountain View Drive, Colchester, VT 05446.
}

Research on the effects of alcohol on automobile driver performance (Moskowitz \& Robinson, 1988), as well as research with pilots (L. E. Ross \& Mundt, 1988), shows a narrowing of the attentional field following even relatively small amounts of alcohol (e.g., blood alcohol concentrations [BACs] near $0.04 \%$ ). Presumably this narrowing reflects a focusing of attention on central tasks in order to compensate for the deleterious effects of alcohol, which could significantly attenuate the apparent effects of alcohol on performance (Gustafson \& Källmén, 1990; D. F. Ross \& Pihl, 1988). An example of such compensation is flying an instrument approach, or in the analogous laboratory situation, keeping two needles representing glide slope and localizer centered. Studies have demonstrated that subjects can do reasonably well at such tasks under low workload conditions, even with relatively high BACs (Chiles \& Jennings, 1970; Pearson, 1968). However, other concurrent and important activities may be ignored and errors committed, as seen in the data of Billings, Wick, Gerke, and Chase (1972), who tested pilots under actual flight conditions. Although their data indicate that alcohol did affect tracking performance, the importance of the effects with respect to flight safety were minimal in some cases, even with elevated BACs. The most interesting data were "major procedural errors" committed by the pilots, which showed a linear increase for both inexperienced and experienced pilots as BAC increased.

Concern with such matters has led to an examination and reconsideration of the way in which the effects of alcohol on performance should be assessed. Consider two kinds of situations that might be evaluated. The first might be called defined task scenarios, in which the primary task 
is to perform a well-known and practiced task, such as flying an instrument approach. Such performances allow deviations from an ideal path to be identified and evaluated easily. However, operators may be able to compensate for a diminished capacity by shifting attention away from other important peripheral tasks. Thus, such tasks may underestimate the effects of alcohol, and effects that are statistically significant may be of such small magnitude that they are of little practical significance with respect to overall system safety. Two variations of the defined task paradigm can improve the evaluation process: (1) introduce additional realistic tasks requiring operator attention such that concentration on a single task is not possible, and (2) apply performance models that integrate multiple performance measures and are sensitive to compensatory attentive behavior (Leirer, Yesavage, \& Morrow, 1989; Morrow, Leirer, \& Yesavage, 1990; L. E. Ross \& Mundt, 1988).

A second type of situation that can be used to evaluate alcohol or drug effects uses episodic task scenarios, in which specific situations may be embedded in realistic operational contexts. Such scenarios present operators with unexpected situations during the course of normal, routine system control in which attention to multiple variables, the integration of information, and a correct sequencing of responses are required simultaneously. Aviation examples of such situations might include carburetor icing, unintentional flight into clouds, or recovery from unusual attitudes presumably due to air turbulence. In a driving simulator, such events might include erratic driving of other vehicles, objects entering the roadway, or a flat tire. Such situations may be more sensitive to the effects of alcohol and other drugs since the events cannot be anticipated and appropriate responses depend upon awareness of a wider variety of system variables. Scoring such scenarios, however, is complicated by the unique circumstances surrounding the occurrence of the specific events.

A further distinction that has been found useful is that of time-driven versus event-driven schedules. In the former, the events in the testing scenario are time predetermined and initiated by an experimenter or a computer program, thus standardizing the sequencing and timing of critical events for all subjects. Given the variability between and within operators, however, the time at which comparable circumstances will obtain during two different testing sessions such that a specific event can be initiated may not be predictable. Consequently, comparison of the responses to seemingly identical events may not be possible because of differences in the situations during which the events occurred. In contrast, event-driven schedules allow experimenters to initiate specific scenario events to accommodate operational situations as they develop. The emphasis for event-driven scenarios is the standardization of the environment at the time particular events are initiated, rather than requiring the sequencing and timing between events to be identical across testing sessions.

Problems may arise in the scoring of event-driven schedules because of idiosyncratic responses or unusual errors made by subjects prior to the initiation of planned events. Such responses or errors, which may be particularly likely under the influence of alcohol or other drugs, may cause or contribute to qualitatively different operational situations, and a priori measures intended to serve as performance indices may be inappropriate or nonapplicable for a specific instance. In such cases, performance evaluation requires a flexible means of viewing and scoringboth qualitatively and quantitatively - various performance parameters that might have been of little interest had anticipated responses occurred. Although it is possible to program conditional evaluation algorithms, based on identification of specific system/situational contexts, in automated performance analysis software, it is virtually impossible to anticipate the range of performance deviations that often occur with intoxicated subjects. Development of procedures designed to evaluate unique events in analysis programs, post hoc, to evaluate data resulting after unanticipated events have occurred, is time-consuming and cost ineffective. A more elegant solution is to provide an interactive graphical analysis package that allows the data analyst freedom to scan and quantitatively measure relevant performance parameters at user-specified spatial and temporal resolutions. Presented below are an example of an automated performance evaluation of an episodic flight event that occurred as planned and an example of the use of an interactive graphical program that permitted analysis of a unique and unanticipated pilot error.

\section{Data Collection}

A modified Frasca 141 flight simulator was used to collect the data for the examples presented below. An Iris2400-based visual system permitted other air traffic to be shown and visibility conditions to be varied. The visual graphics were projected on a screen $1.9 \mathrm{~m}$ wide by $1.4 \mathrm{~m}$ high located $2 \mathrm{~m}$ in front of the pilot. The geographic area was that of the San Francisco Bay area with several airports, differing in runway width and length, included.

Collection and storage of data were controlled by an IBM PC with a serial port connected to the Frasca computer, $3 \mathrm{MB}$ of RAM, and three parallel ports and $16 \mathrm{~A} / \mathrm{D}$ channels connected to flight instruments in the simulator. A time-encoded view of the flight instruments was videotaped during the flight sessions, and subject-controller communications were tape recorded to provide for the checking of data records during any point in the flight scenario. The experimenters (flight or ground instructors) served as air traffic controllers, creating realistic flight situations and initiating appropriate flight events during the scenarios. An "experimenter box" with numbered buttons that could be pressed was also sampled and stored by the data-collection computer, providing specific time marks in the data record to facilitate the event analyses presented below.

\section{Episodic Flight Event Scoring Example}

An example of scoring a pilot's response to a towerinitiated "go-around" is presented below. As the pilot 
Table 1

Abbreviated Analysis of Go-Around Event Performance

Go-around event initiated at $1,343.7 \mathrm{sec}$

Carb heat off at $1,602.4 \mathrm{sec}$

$\mathrm{RT}=258.7$

Flaps up at $1,755.9 \mathrm{sec}$

$\mathrm{RT}=412.2$

Initial throttle setting $=0.150$

Throttle up to 0.840 at $1,345.2 \mathrm{sec}$

$\mathrm{RT}=1.5$

$\begin{array}{ccc}\text { Initial conditions } & \text { Minimum } \\ \text { Airspeed } & 61.23 & 60.84\end{array}$

$\begin{array}{lrr}\text { Altitude } & 212.13 & 203.09\end{array}$

\author{
Turn initiated at $1,345.4 \mathrm{sec} \quad$ RT $=1.7$ \\ Level off at $1,179.08 \mathrm{ft}$ at $1,453.6 \mathrm{sec}$ \\ Initial altitude $=212.13$ \\ Initial airspeed $=63.02$; Average airspeed $=80.44$ \\ Max slip $=0.46 ;$ Average slip $=0.095$
}

reached the runway threshold, control-tower instructions were given to initiate an immediate go-around, to execute a climbing left or right turn to a specific heading, and to level off at $1,000 \mathrm{ft}$. A button on the experimenter box was pressed when the pilot was given the instructions, when the turn was initiated, and when the pilot reached $1,000 \mathrm{ft}$ or level flight.

The pilot's response was scored automatically by computer, and relevant performance data were written to summary files for further analyses. Relevant event times were read directly from the parallel-port data file, as were the times associated with raising the flaps and turning off carburetor heat. Control inputs and flight parameters such as altitude, airspeed, and bank angle were available, given the time of event initiation and the sampling frequency of the channels.

An abbreviated analysis of a pilot's responses to such an event is shown in Table 1, where it can be seen that the pilot's response was full throttle and immediate execution of the turn. No attempt was made to retract the flaps, turn off the carburetor heat, or gain altitude before executing the turn, which would have been procedurally correct. The turn was executed fairly well, however, as indicated by the lack of any significant loss of altitude or airspeed and the moderate slip indications. Other measures in the analyses not included in Table 1, such as pitch, roll, and turn rates, were within acceptable limits.

Such scoring procedures permit automated and detailed performance analysis of these types of event-driven, episodic scenarios-provided that the experimental scripts designed to investigate particular aspects of operator performance are properly executed. Creating comparable situations for the initiation of specific events requires operators to comply with directives given by the experimentercontroller within predictable limits. Idiosyncratic responses due to misunderstandings or misinterpretations may occur, however, and these unexpected responses may be particularly likely under the influence of alcohol or other drugs. Such occurrences, which may represent operator error in judgment or maneuver execution, can qualita- tively alter the ensuing performance environment, preventing the realization of readily comparable situations for the initiation of subsequently planned events. These differences may be of equal or greater importance with respect to system safety than the quantifiable differences in response to planned events, but the significance of these incidents may not be recognized without a readily available means for analyzing them.

Thus, in addition to scoring episodic events as described above, there is also a need for a flexible analysis system not dependent upon experimenter-controller-identified time marks for the analysis of simulator performances. Interactive graphical analysis allows flexible scoring of performance in situations where unanticipated operator behaviors may prevent routine performance evaluation from being accomplished.

\section{Interactive Performance Analysis}

The system described below for the analysis of data obtained under such circumstances is a menu-driven, PC-based graphics program developed and written in Turbo Pascal (Version 4) that makes extensive use of the graphics toolbox. The flight path followed by a pilot can be viewed on a variable-resolution map, and stored performance parameters such as altitude, airspeed, and control inputs can be plotted across user-specified time bases. The program allows an analyst to view an entire flight path or performance parameter on the monitor screen over the entire performance period or to "zoom in" on segments of flight that hold specific interest. By plotting multiple flight parameters over common time bases, a complete performance profile can be reconstructed, interpreted, quantifiably measured, and analyzed. The analyst can use pattern-recognition skills and domain-specific knowledge of the task environment to gain insight into aspects of operator performance and system safety that might be missed or uninterpretable given only the type of numerical summary data shown in Table 1.

Presented below is an example demonstrating how a presumably alcohol-induced error can lead to difficulties with predefined scoring systems, and how interactive graphical analysis can be used to understand unexpected event sequences. On each testing day, the pilot was directed by air traffic control (ATC) to an approach course at an assigned altitude that should have been maintained until passing a final approach fix (a spatial location identifiable using navigational instruments). The pilot was then expected to descend, maintaining a constant airspeed and descent rate, for a specific period of time (indicated on the published approach plate for the particular airport) until establishing visual contact with the runway (a "nonprecision" approach). If visual contact was not established within the time allotted on the approach plate, a missed approach was to be executed and the pilot was to proceed to an alternative runway. Using the previously described scoring procedures, the experimenter would time mark the data record at specific points of interest during the approach, and these marks would allow quantitative scor- 
ing of parameters such as altitude, airspeed, and vertical velocity during the different segments of the approach.

Figure 1 is a plot of the final 5 min of flight on the day that the pilot ingested no measurable quantity of alcohol (placebo condition). Numbered triangles mark the number of minutes into the flight; San Jose (SJC) airport is shown in the lower right corner; and SUNNE (SUN) intersection (approach fix) is marked by an $\mathrm{X}$ between Minutes 57 and 58. An X surrounded by a box and indicated by an arrow is at 57:57 into the flight and represents the location at which the pilot throttled back and began his descent. Figure 2 shows the plots of altitude, airspeed, throttle position, and aircraft pitch profiles from the beginning of Minute 56 to the end of Minute 60 . It can be seen that the pilot reduced throttle just before Minute 58 (57:57 was found by zooming in on Minute 57), decreased pitch, maintained a steady 90 -knot descent, and proceeded to land. In this instance, events occurred as expected and the performance could easily be evaluated with respect to an idealized flight path.

Figure 3 is the flight path, starting from the lower right part of the graph, taken by the aircraft on the day the pilot received alcohol (.039\% BAC entering, .026\% BAC exiting the simulator) and committed a very serious and unexpected error. The pilot was directed on to the approach course to Hayward airport (HAY, upper left) between the IMPLY (PLY, lower right) and DECOT (DCT, center) navigational intersections at about Minute 34 . It was expected that he would maintain the altitude assigned by ATC to the approach fix DECOT (which was the min-

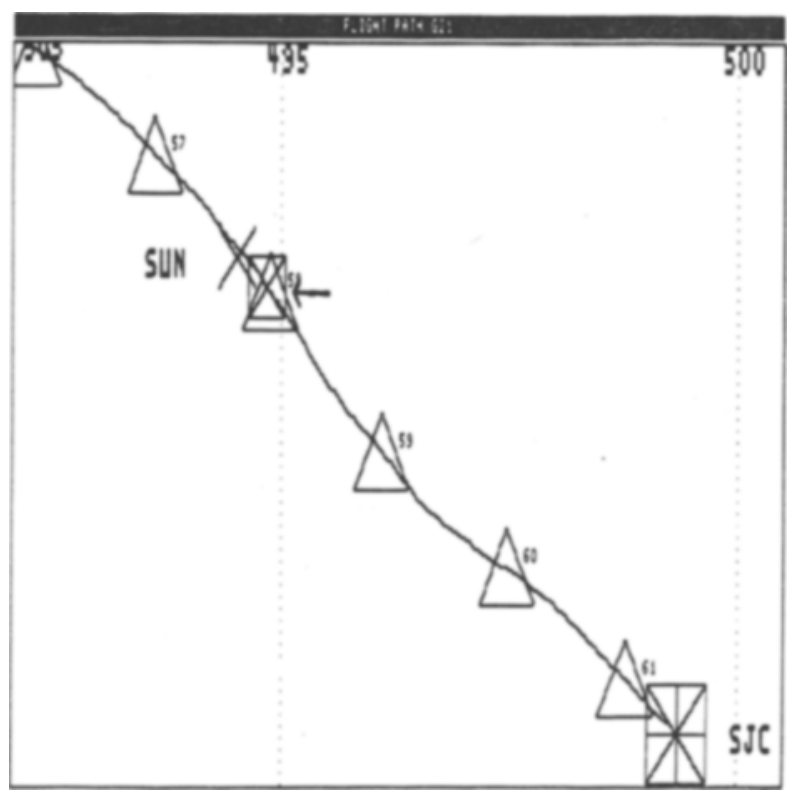

Figure 1. Flight path of simulator on approach to San Jose (SJC). Numbered triangles represent location at that minute into the flight, SUN $(X)$ indicates location of approach plate intersection from which descent should begin, and the $X$ surrounded by $a$ box and indicated by an arrow shows location at which descent began.

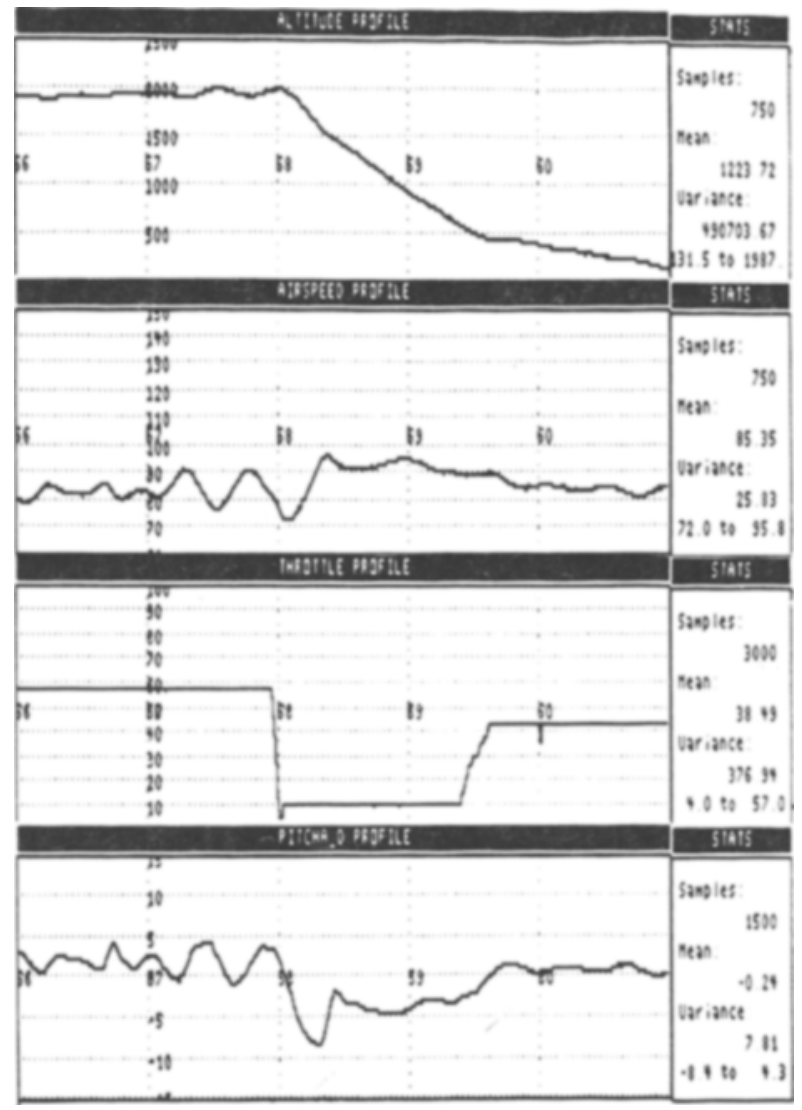

Figure 2. Flight parameter plots of altitude, airspeed, throttle position, and aircraft pitch for Minutes 56-61 during approach to San Jose.

imum allowable altitude at that location, as indicated on the approach plate for Hayward) and then execute the nonprecision approach (maintaining 90 knots) to the runway. Figure 3 shows that the pilot unexpectedly turned away from Hayward and executed a missed approach while still several miles short of the airport. Figure 4 shows the same flight-parameter plots for this flight segment as those shown in Figure 2 . The first feature that should be noted is that the pilot completed his descent and was already at minimum descent altitude for Hayward airport by the time he reached the final approach fix DECOT (near Minute 36). Second, it can be seen in the throttleposition profile that the missed approach was executed midway between Minutes 37 and 38 (37:22). This position is indicated in Figure 3 by an arrow pointing to an $\mathrm{X}$ surrounded by a box.

After completion of the flight, the pilot was debriefed concerning his performance. He indicated that he had timed his approach and executed a missed approach at 3:03 after descent initiation, which is consistent with the time printed on the Hayward approach plate to execute a missed approach for a $90-k n o t$ descent. The pilot also reported difficulty maintaining a steady airspeed during the approach. Since the missed approach was executed 
at 37:22, the pilot must have begun timing at $34: 19$, and the location of the aircraft at that time is indicated in Figure 3 near IMPLY. The altitude profile in Figure 4 shows that it was near this time that the pilot began his descent, and the airspeed profile confirms the pilot's retrospective report of poor airspeed control during the descent.

This type of interactive analysis allows the performance analyst to reconstruct the flight event in detail and to gain insight into a serious error that was unexpected and somewhat bewildering to the experimenters at the time of its occurrence. Although the pilot was at the assigned altitude of $2,600 \mathrm{ft}$ and directed by ATC to intercept the approach path to Hayward between IMPLY and DECOT, it is clear that he mistook the first intersection for DECOT and executed his timed descent at that point. The result was that he was at minimum descent altitude by the time he reached DECOT, the time at which the descent should have begun. Review of the videotaped episode indicates that the pilot was never aware of the error, either during or after the event.

Unexpected pilot responses or errors encountered in the standardized, time-marked event analysis can be analyzed without an interactive program of this type, but dealing with each unique performance via post hoc programming is expensive and time consuming. With an interactive graphical analysis technique, it is relatively fast and effortless to reconstruct and analyze (both quantitatively and qualitatively) unanticipated events that occur at any time during the testing scenarios.

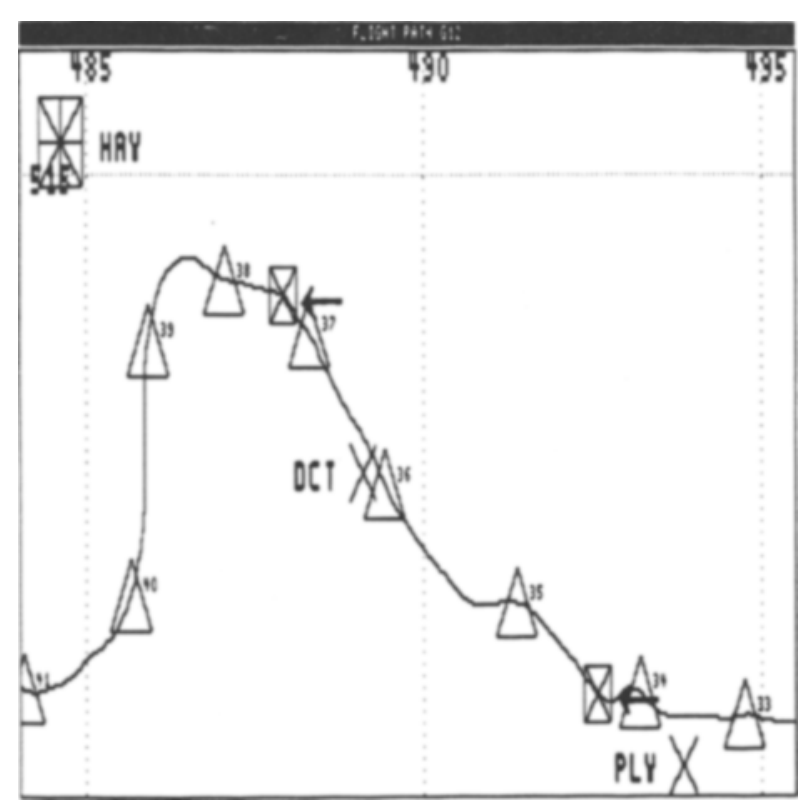

Figure 3. Flight path of simulator on approach to Hayward (HAY). Numbered triangles represent location at that minute into the flight, DCT (X) and PLY (X) indicate location of approach plate intersections, $X$ surrounded by a box between Minutes 34 and 35 indicates location where descent began, and $X$ surrounded by a box between Minutes 37 and 38 indicates location where missed approach was executed.

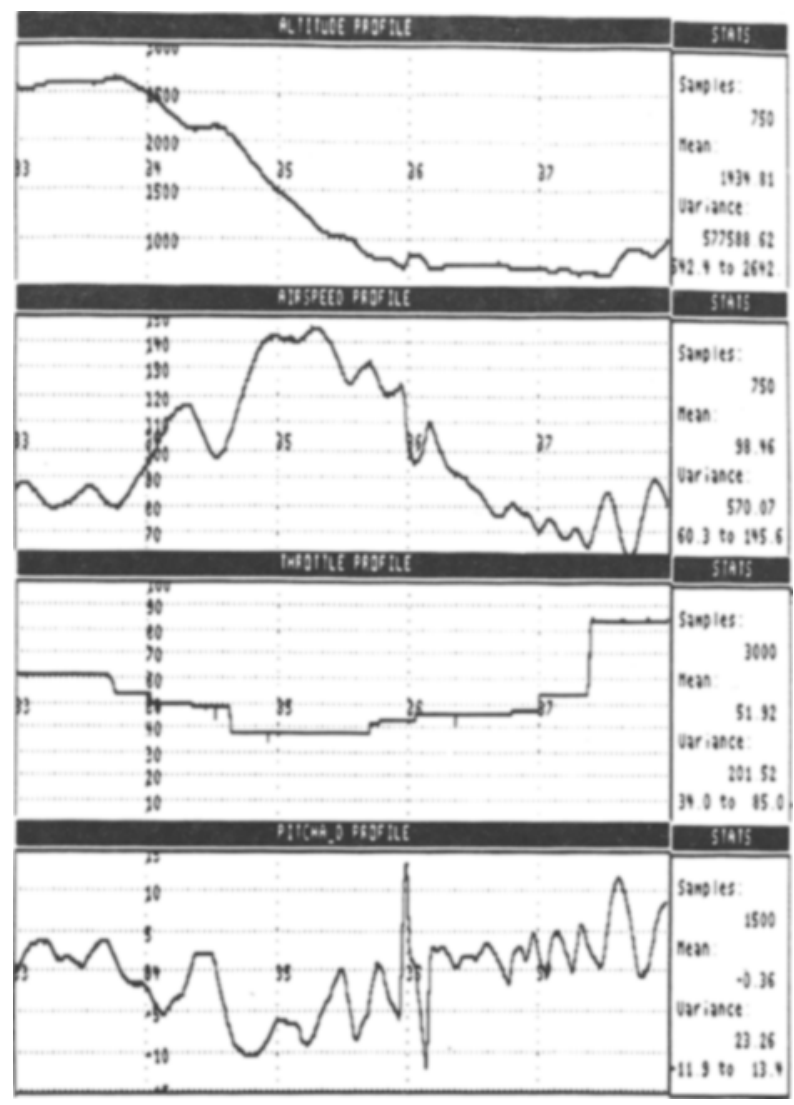

Figure 4. Fight parameter plots of altitude, airspeed, throttle position, and aircraft pitch for Minutes 33-38 during approach to Hayward.

\section{Discussion}

Large individual differences and the idiosyncratic manner in which alcohol and other drugs affect performance present challenges to researchers concerning how testing situations should be structured to evaluate their effects. Defined task scenarios use tasks for which the requirements are well known to the operators and allow relatively simple comparisons to be made automatically with respect to idealized performance profiles. Such scenarios are useful for evaluating performance decrements for specific tasks, but operators may be able to allocate attentional resources such that the true effects of alcohol or other drugs are underestimated. Episodic scenarios, on the other hand, are characterized by distinct, sometimes unexpected, events embedded in normal operational contexts. These types of scenarios allow a more functional assessment of performance and are thus less likely to be sensitive to the resource-allocation strategies employed during experimental sessions.

Fine-grained analysis of event-driven scenarios depends upon the initiation of common events during similar testing contexts across different experimental sessions and the identification of these events in the data record. How- 
ever, subjects' idiosyncratic drug or alcohol responses may preclude the attainment of easily comparable situations in which to introduce the same events in both the drug and no-drug conditions. Thus, flexible analytic procedures for assessing performance are required. Interactive performance analysis, based on graphical viewing and measurement of performance parameters, allows analysts to scan through considerable data efficiently and to identify and focus on unique features of the recorded performance. Using pattern-recognition skills and domainspecific knowledge, performance analysts can reconstruct, interpret, and understand idiosyncratic responses that may be extremely important in understanding compromised system safety.

The example provided here pertains specifically to the analysis of pilot performance in a simulated flight environment, but the interactive graphical approach to data analysis is applicable to many experimental simulations. Although this analytic approach is particularly relevant to testing situations in which subjects have sufficient behavioral latitude to effect divergent consequences, such as system simulations, it is useful in any situation in which highly individualistic responses to experimental manipulations are likely. Interactive viewing and graphical measurement of performance data is currently being applied to such divergent aspects of alcohol-related performance as oculomotor functioning, analysis of speech production (Perrine, Krüger, \& Kelleher, in press), and postural control and coordination during locomotion. The flexibility to examine data at user-specified resolution, to compare performance indices of specific interest to situational contexts, and to quantify measurements from these observations allows insight into idiosyncratic effects of alcohol and other drugs that may be missed if more rigid, preplanned performance measures based on a priori assumptions are applied exclusively. As behavioral-testing situations more closely approximate naturalistic environments, constraints on possible responses by subjects to specific circumstances diminish. As the potential for eliciting divergent and individualistic responses increases, the need for versatile analytic methods for performance assessment increases.

\section{Program Availability}

Although the program described for analyzing idiosyncratic pilot performance is specific to the data records and formats developed for the flight simulator described, the Turbo Pascal source code incorporating the Graphics Toolbox procedures can be obtained by sending a formatted high-density disk to the first author or by sending a request to jmundt@uvmvax.uvm.edu.

\section{REFERENCES}

Billings, C. E., Wick, R. L., Gerke, R. J., \& Chase, R. C. (1972). The effects of alcohol on pilot performance during instrument flight (Rep. No. FAA-AM-72-4). Oklahoma City: Civil Aeromedical Institute.

Chiles, W. D., \& Jennings, A. E. (1970). Effects of alcohol on complex performance. Human Factors, 12, 605-612.

Gustafson, R., \& KällmÉN, H. (1990). Alcohol and the compensation hypothesis: A test with cognitive and psychomotor tasks. Perceptual \& Motor Skills, 71, 1367-1374.

Leirer, V., Yesavage, J., \& Morrow, D. (1989). Marijuana, aging. and task difficulty effects on pilot performance. Aviation, Space, \& Environmental Medicine, 60, 1145-1152.

Morrow, D., Leirer, V., \& Yesavage, J. (1990). The influence of atcohol and aging on radio communication during flight. Aviation, Space, \& Environmental Medicine, 61, 12-20.

Moskowitz, H., \& RoBinson, C. D. (1988). Effects of low doses of alcohol on driving-related skills: A review of the evidence (DOT-HS-807280). Washington, DC: National Highway Traffic Safety Administration.

Nolan, M. D., Hettinger, L. J., Kennedy, R. S., \& Edinger, K. M. (1988). Individual differences in flight simulation performance experiments. Proceedings of the 32nd Annual Meeting of the Human Factors Society, 2, 1001-1005.

Pearson, R. G. (1968). Alcohol-hypoxia effects upon operator tracking, monitoring, and reaction time. Aerospace Medicine, 39, 303-307.

Perrine, M. W., Krüger, H. P., \& Kelleher, P. F. (in press). Speech chronemics and alcohol: New dimensions of an ancient relationship. Alcohol Health \& Research World.

Ross, D. F., \& PIHL, R. O. (1988). Alcohol, self-focus, and complex reaction-time performance. Journal of Studies on Alcohol, 49, 115-125.

Ross, L. E. \& MUNDT, J. C. (1988). Multiattribute modeling analysis of the effects of a low blood alcohol level on pilot performance. Human Factors, 30, 293-304.

(Manuscript received March 3, 1992; revision accepted for publication January 20, 1993.) 